\title{
Novel m-PSO-SVM based Interface Controller Design for Haptic System
}

\author{
Naveen Kumar, Student Member, IEEE, and Jyoti Ohri, Member, IEEE
}

\begin{abstract}
The haptic system has two key performance issues: stability and transparency. A haptic interface controller (HIC) is designed to address these issues. Addressing these issues becomes a complex problem as both are complementary to each other. Here, when transparency of the system is increased, its stability degrades and vice-versa. To overcome this problem, intelligent optimized solutions are used in this paper to design a HIC controller for the haptic system. SVM and NN techniques have been employed to identify the performance of the controller, ensuring stability and transparency both. The disadvantages of $\mathrm{NN}$ in terms of the number of neurons and hidden layers are overcome by SVM. Further, the performance of SVM is highly dependent upon the selection of free parameters. So, further, a modified PSO technique is employed for the optimal selection of these parameters to enhance the performance of SVM. Hence, this novel proposed hybrid technique of m-PSO optimized SVM is applied for the optimal design of the HIC to find out an optimal solution between trade-off the transparency and stability of the haptic device simultaneously. To appreciate the efficacy of the proposed technique, the result obtained with this is compared with HIC design using neural network and conventional ZN method also. This designed controller ensures stability as well as transparency, even under the presence of uncertainty, delay, and quantization error.
\end{abstract}

Index Terms-Haptic system, stability, transparency, neural, SVM, m-PSO-SVM, HIC.

\section{INTRODUCTION}

$\mathrm{H}$ APTIC system is the combination of hardware and software so connected that the user feels as if he is interacting with the real objects using haptic feedback techniques. This feedback can be in terms of either force, velocity, sound or vibration, etc. The applications of this technique include the training of medical surgeons and the pilot before hands-on with the real body or system. To ensure that the user feels realistic objects while interacting, there should be minimum deviation between the applied user force or velocity and the one executed in the virtual environment (VE) [1]. This minimization in error is termed as transparency. Further, the stability of haptic system may be defined as how fast oscillations or vibrations in the output response of the system

Manuscript received January 18, 2020; revised May 11, 2020. Date of publication June 4, 2020. Date of current version June 4, 2020.

Authors are with the Electrical Engineering Department, NIT Kurukshetra, Haryana India, India.

E-mails: naveenvermaindia@gmail.com, ohrijyoti@ rediffmail.com.

Digital Object Identifier (DOI): 10.24138/jcomss.v16i2.1002 get settled over a definite period of time. In literature, several authors have worked on the above said two performance issues but not simultaneously [2].

Passivity based approach has been used by Weir et. al in [3] which consider user end as a passive port for keeping system stable. In [4], [5] Colgate et al., explain the passivity condition, which includes VE, interface controller, and the haptic device. Routh Hurwitz method has been used to find the boundary limits on HIC parameters for stability. The stability range is also defined for LHIfam haptic system under the presence of delay in [6]. Further, various factors affecting the performance issues are required to be considered in the model to make it a more realistic one while designing the controller. Transparency can be maximized by minimizing the error between applied user force and the feedback force from VE. Transparency and stability are complementary to each other. Maximizing the transparency will minimize the stability and vice-versa [7]. So optimal solution is required so as to maintain balance between achieving the stability of the system along with improved transparency.

Over the period of time, various soft computing techniques have been evolved, such as genetic algorithm, neural network, particle swarm optimization, fuzzy logic, etc. [8]-[13]. These techniques have shown various advantages and disadvantages of different applications. An optimization algorithm (OA), which has given the best results for an application, may or may not provide an optimal solution for others. The neural network has proved its competency in a wide range of applications in literature [14]-[17]. But it has been found that NN is prone to local convergence, slow learner, and overfitting problems in some applications [16]. Recently, support vector machine (SVM) has emerged as a tool to overcome the drawbacks of NN [11], [18], [19]. The SVM has three independent factors that affect its performance significantly. The selection of these parameters is generally by TAE method and prior expertise knowledge [20]. The selected factors of SVM for one application sometime may not fit for others. Moreover, if one has to apply this tool for other applications or uncertainty is added to the system, then these parameters have to be tuned again. This application-specific selection creates more difficulties and time-consuming process for the researcher always. The learning and regression properties of SVM have made it suitable for identifying the parameters of the HIC controller. The independent parameters of SVM, which are to 
be chosen by the designer, impact the performance of SVM.

In this paper, a novel hybrid intelligent technique is proposed. This technique employs modified PSO for the optimal selection of SVM parameters to optimize its performance. Further, modification in PSO also ensures the faster convergence to global minima than its precedent. The results obtained using this proposed hybrid technique is compared with HIC design by classical technique ZN method and NN.

The organization of this paper is as follows. Section II describes a neural network, SVM, and proposed m-PSO optimized SVM. The problem model of the haptic system is formulated in section III. The haptic interface controller (HIC) design algorithm and simulation results using different optimizing techniques are given in section IV. The comparison of results is discussed in section $\mathrm{V}$, followed by the conclusion section.

\section{CONTROL DESIGN TECHNIQUES FOR HIC}

To ensure the transparency and stability of the haptic device, so that the tradeoff between the two is taken care of, the optimal solution of parameters of HIC is required. In this section techniques such as NN, SVM and proposed m-PSO optimized SVM are presented which are employed for the optimal design of HIC in this paper.

\section{A. Neural Network}

The interconnections of neurons are known as neural network. In this, neurons are the combination of summing node and activation function. These are connected using weights and adjusted using different training techniques and the desired outputs. There are ' $m$ ' number of inputs connected to ' $n$ ' number of neurons [21]. The weight matrix ' $w$ ' and input vector ' $x$ ' given in (1) and (2) respectively.

$$
\begin{array}{r}
x=\left[x_{1} x_{2} x_{3} \ldots \ldots . x_{m}\right]^{T} \\
\mathrm{w}=\left[\mathrm{w}_{1}, \mathrm{w}_{2}, \mathrm{w}_{3}, \ldots . . \mathrm{w}_{\mathrm{m}}\right]
\end{array}
$$

The output function ' $y$ ' may be defined as

$$
y=f\left[\left(x_{1} w_{1}+x_{2} w_{2} \ldots \ldots x_{m} w_{m}\right)+b\right]
$$

where $b$ is the bias. The mathematical expression for neurons is defined as

$$
y=f(x)=f\left[b+\sum_{k=1}^{m}\left(x_{k} w_{k}\right)\right]
$$

Now, the output vector for ' $y$ ', defined as

$$
y=\left[\begin{array}{llllll}
y_{1} & y_{2} & y_{3} & \ldots & \ldots & y_{n}
\end{array}\right]^{T}
$$

The input and the output vector can be termed as patterns. Here, the $p^{\text {th }}$ neurons would be connected to the $q^{\text {th }}$ output using the weight $w_{p q}$. The output of each neuron as

$$
a_{p}=\sum_{q=1}^{m} w_{p q} x_{q} \text { for } p=1,2,3 \ldots \ldots n
$$

The $p^{\text {th }}$ neuron output with activation function can be written as

$$
y_{p}=f\left(w_{p}^{t} x\right) \text { for } p=1,2,3 \ldots . m
$$

The weight vector $w_{p}$, is the weight linked with $p^{\text {th }}$ neurons can be written as

$$
w_{p}=\left[w_{p 1} w_{p 2} \ldots w_{p m}\right]
$$

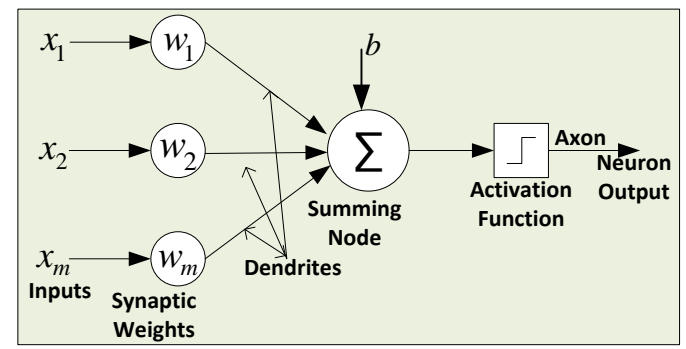

Fig. 1. Artificial Neurons Structure

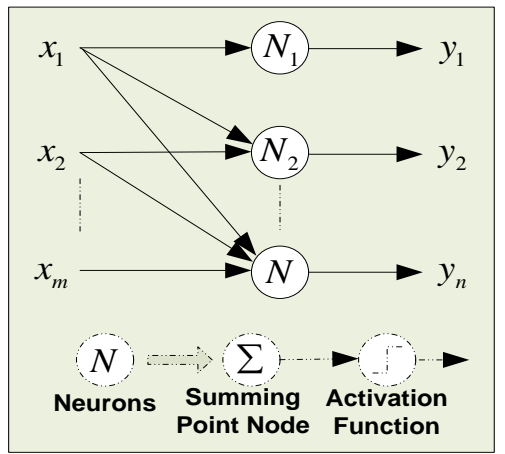

Fig. 2. Neurons architecture for Feed-forward network

\section{B. Support Vector Machine (SVM)}

The SVM was introduced by Vapnik et al [22] received popularity in a short period of time. It uses a supervised learning method to overcome the disadvantages of neural network (NN), to select the number of neurons and the hidden layers for different systems. Further, it has shown regression in limited samples for various applications. The simpler form of SVM is linear SVM shown in Fig. 3 having a single line separating the data represented in (9) [23].

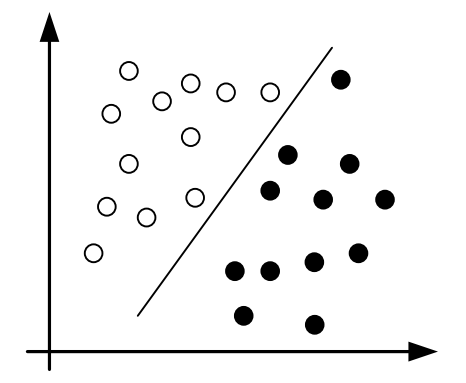

Fig. 3. Separation of data set with L-SVM

Further, for better prediction, the width of the separating line has been increased as shown in Fig 4 . 


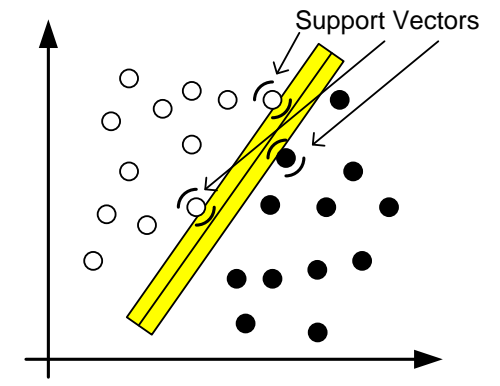

Fig. 4. Widen separating line and support vectors

The data points touching this widen line, are known as support vectors. The width of the separating line can be increased within a certain limit, as given in the equation below.

$$
\begin{gathered}
w_{s v m} \cdot x+b \leq 1 \\
w_{s v m} \cdot x+b \geq-1 \\
-1 \leq\left(w_{s v m} \cdot x+b\right) \leq 1
\end{gathered}
$$

where $\mathrm{x}$ is the input data, $\mathrm{w}_{\mathrm{svm}}$ and $\mathrm{b}$ are constants. This limit helps while selecting the data points, and based on this, a linear function may be rewritten as (12).

$$
f(\mathrm{x})=\left\langle\mathrm{w}_{\text {svm }}, \mathrm{x}\right\rangle+\mathrm{b}
$$

where $f(x)$ is an unknown target function and $\langle.,$.$\rangle represents$ the inner product. Now to generalize this method, $\left\{\mathrm{x}_{1}, \mathrm{y}_{1}\right\}$, $\left.\left\{\mathrm{x}_{2}, \mathrm{y}_{2}\right\} \ldots \ldots . . \mathrm{x}_{1}, \mathrm{y}_{1}\right\}$ are considered as input data set such that $\mathrm{X} \in \mathbb{R}$ where ' $\mathrm{X}$ ' represents the for input space and $\mathrm{Y}_{1} \in$ $\{-1,1\}$. Moreover, a soft margin $\xi$ is also taken into account while considering the data for better prediction, as shown in Fig 5 .

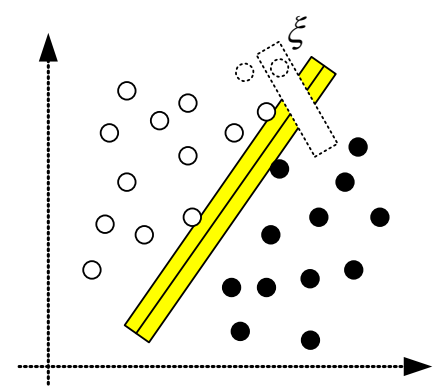

Fig. 5. Inclusion of soft margin into the separating line

The generalized formula for SVM including the soft margin is as follows:

$$
\text { Minimize } \frac{1}{2}\left\|w_{\text {svm }}\right\|^{2}+C \sum_{i=1}^{l}\left(\xi_{i}+\xi_{i}^{*}\right)
$$

Subject to

$$
\left\{\begin{array}{c}
y_{i}-\left\langle w_{s v m}, x_{i}\right\rangle-b \leq \varepsilon+\xi_{i} \\
\left\langle w_{s v m}, x_{i}\right\rangle+b-y_{i} \leq \varepsilon+\xi_{i}^{*} \\
\xi_{i}, \xi_{i}^{*} \geq 0
\end{array}\right.
$$

Here, constant $C>0$, used to determine the trade-off between the flatness ' $f$ ' and deviation amount accepted larger than ' 1 '. This is called as insensitive loss function $\xi$ and it can be represented as

$$
|\xi|= \begin{cases}0, & \text { if }|\xi|<1 \\ |\xi|-1, & \text { otherwise }\end{cases}
$$

Using the langrage multiplier, and kernel techniques in [24]

$$
\begin{gathered}
L=\min _{\propto i, \alpha^{*}}\left(\frac{1}{2} \sum_{r, s=1}^{l}\left(\propto_{r}^{*}-\propto_{r}\right)\left(\propto_{s}^{*}-\propto_{s}\right) * K\left(x_{r}-x_{s}\right)-\right. \\
\left.\sum_{r=1}^{l}\left(\propto_{r}^{*}-\propto_{r}\right) y_{r}+\varepsilon \sum_{s=1}^{l}\left(\propto_{s}^{*}-\propto_{s}\right)\right)
\end{gathered}
$$

Subject to

$$
\sum_{r=1}^{l}\left(\propto_{r}^{*}-\propto_{r}\right)=0
$$

where $0 \leq \propto_{r}, \propto_{r}^{*} \leq C, \mathrm{r}, \mathrm{s}=1 \ldots .1$.

Further, radial bias krnel function based nonlinear SVM technique [25], [26] can be formulated as

$$
K\left(x_{r}-x\right)=\exp \left(-\gamma\left|x-x_{r}\right|^{2}\right.
$$

And using KKT condition

$$
b= \begin{cases}y_{r}-\sum_{s=1}^{l}\left(\propto_{s}^{*}-\propto_{s}\right) * K\left(x_{r}-x_{s}\right)+\varepsilon & \propto_{r}>0 \\ y_{r}-\sum_{s=1}^{l}\left(\propto_{s}^{*}-\propto_{s}\right) * K\left(x_{r}-x_{s}\right)+\varepsilon & \propto_{r}^{*}>0\end{cases}
$$

Using this equation, SVM output would be

$$
\hat{\mathrm{f}}(\mathrm{x})=\sum_{s=1}^{l}\left(\propto_{s}^{*}-\propto_{s}\right) * K\left(x_{r}-x_{s}\right)+\bar{b}
$$

where $\bar{b}$ represents average of $b$ using (17).

The error of the system should be

$$
|\hat{f}(x)-f(x) \leq \varepsilon|
$$

The SVM model based on RBF kernel has three factors name as cost factor $(C)$, gamma $(\gamma)$ and epilson $(\varepsilon)$ which are to be selected using TAE and research experiences. Further, the selection of these factors affects the performance of this technique for various applications. Manual selection of three free parameters is very time consuming and also applicationspecific. Hence need was felt to find a solution so that automatically optimized values of these parameters can be found.

\section{Proposed m-PSO Optimized SVM}

It is proposed in this paper that a modified PSO can be introduced for the optimal selection of free parameters of SVM. The detail is given below.

\section{1) Modified Particle Swarm Optimization (m-PSO)}

The PSO concept was introduced by J. Kennedy et. al. in [27]. It is a population-based search algorithm in which the particle converges to find the best optimal solution within a given search space. Each particle moves with a velocity that dynamically changes according to particle's previous best experience and along with its neighborhood best position in the previously visited search space. Velocity and position of each particle are updated with population law locally in pbest and globally gbest. Velocity and position update formulas are given 
in (19) and (20), respectively [28].

$$
\begin{aligned}
& v_{i, j}^{(k+1)}=w c * v_{i, j}^{(k)}+c 1 * \operatorname{rand}(1) *\left(\text { pbest }_{i, j}-x_{i, j}^{(k)}\right)+ \\
& c 2 * \operatorname{rand}(2) *\left(\text { gbest }_{j}-x_{i, j}^{(k)}\right) \\
& x_{i, j}^{(k+1)}=x_{i, j}^{(k)}+v_{i, j}^{(k+1)}
\end{aligned}
$$

There are different modifications introduced by various researchers over time in basic PSO algorithms. The selection of weight ' $w c$ ' would help in a quick search of optimal results in the population. In [28], [29], the idea of variable weight is presented. Further, modification in variable weight factor ' $w c$ ' was presented by [30] as (21). This modification also reduces the local search time and the total time of convergence.

$$
w c=\left(w c_{\max }-w c_{\min }\right)-\left(\frac{\text { iter }_{\max }-\text { iter }}{\text { iter }_{\max }}\right) * w c_{\min }
$$

where $w c_{\max }$ and $w c_{\min }$ are maximum and minimum inertia weights selected as 0.9 and 0.4 respectively, iter represents the current iteration and iter $_{\max }$ denotes the maximum iteration. Initially, inertia weight has large value but decreases continuously with an increase in the number of iterations.

In addition to this, velocity is modified as proposed in [31], for defining the range of velocity $v_{\max }$ and $v_{\min }$, given in (22) and (23).

$$
\begin{aligned}
& v_{\max }=0.1 *\left(k_{\max }-k_{\min }\right) \\
& v_{\min }=-0.1 *\left(k_{\max }-k_{\min }\right)
\end{aligned}
$$

where $k_{\min }$ and $k_{\max }$ are lower and the upper limits respectively. The initial value of velocity and position are calculated as given in (24) and (25), respectively.

$$
\begin{aligned}
& v_{\text {inital }}=v_{\min }+\left(v_{\max }-v_{\min }\right) * \epsilon_{\text {pso }} \\
& p_{\text {inital }}=v_{\min }+\left(v_{\max }-v_{\text {min }}\right) * \epsilon_{\text {pso }}
\end{aligned}
$$

where $v_{\text {initial }}$ and $p_{\text {initial }}$ are initial velocity and the position, $\epsilon_{p s o}$ is the random number matrix.

\section{2) Novel m-PSO optimized SVM}

Modified PSO ( $m-P S O)$ having variable weights, initial velocity and position is applied to find the optimal values of independent or free parameters of SVM. In SVM, the three independent parameters are: cost factor $(C)$, gamma $\gamma$, and epsilon $(\varepsilon)$. In the previous section in (13) ' $C$ ' the cost factor, has been used as a constant value. It affects the loss value of objective function i.e. error value. More clearly, the greater the value of the cost factor, the greater will be the error. When error is large, SVM is on the verge of over-fitting and when ' $C$ ' is too small, SVM might find inadequate fitting.

Next is epsilon $(\varepsilon)$, which controls the width of $\boldsymbol{\epsilon}$-insensitive zone. If $\epsilon$ is too high, the insensitive zone would have enough margin to include data points, and it leads to unacceptable flat regression. Subsequently, parameter gamma ' $\gamma$ ' represents the RBF kernel width. If it is large, then it will make SVM not flexible enough for complex function approximation, if it's small the SVM tends to overfit to the training data. So, instead of using the predefined value of the various SVM parameters, an optimizing tool ( $m-P S O)$ has been employed in this work for optimal selection of these parameters so as to obtain the optimal performance of SVM.

The flow chart of the proposed algorithm is shown in Fig 6 .

Step 1: Firstly, the range of the parameters of SVM which are to be optimized in defined along with the parameters of the $\mathrm{m}$ PSO.

Step 2: Then $m$-PSO is initialized. The fitness value is evaluated against fitness function.

Step 3: After each iteration, update the position and velocity of each particle until the maximum iteration reached.

Step 4: This final value evaluated is called as optimum value.

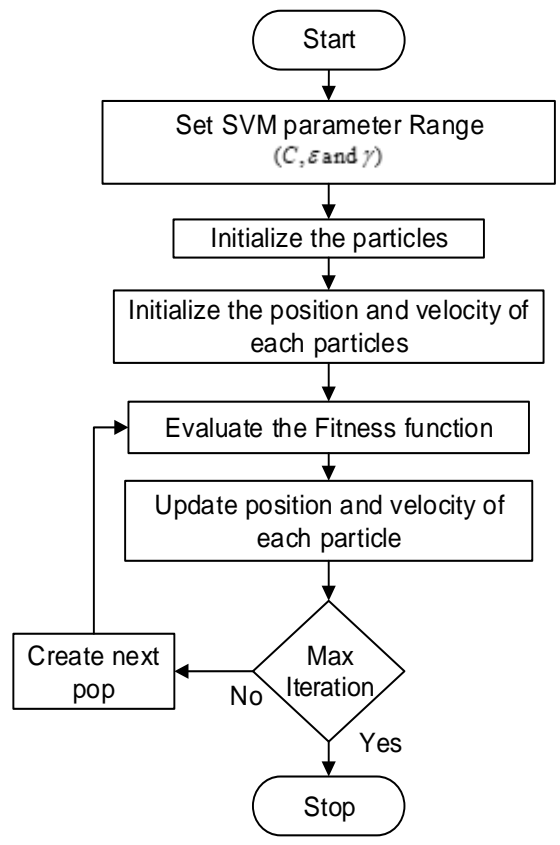

Fig. 6. Flow chart for m-PSO to optimize SVM free parameters 


\section{FORMULATION OF HAPTIC INTERFACE CONTROLLER}

The problem of the design of a continuous-time haptic interface controller (HIC) for the haptic system is taken up in this work. The free body diagram of haptic system is shown in Fig. 7, has been considered is used in this paper, having mass ' $m$ ', damping coefficient ' $b$ '. Assuming ' $x$ ' as position, ' $F$ ' as driving force applied by means of actuating motor and ' $F_{u}$ ' is the user force. The dynamic equation for haptic device can be written as (26)

$$
m \ddot{x}+b \dot{x}=F_{d}+F_{u}
$$

Here, $F$ is considered as net equivalent force, then Laplace transform for this model is

$$
G(s)=\frac{x}{F_{d}+F_{u}}=\frac{1}{m s^{2}+b s}
$$

The corresponding haptic system continuous-time model is shown in Fig 7. This mass damper model is used as a plant in the further text [6].

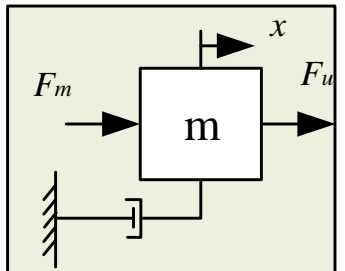

Fig. 7. Haptic system continuous-time model

Further, a complete haptic system is consists of three main building blocks: robotic manipulator, virtual environment (VE) and the interfacing network. The manipulator is used for handling or interaction with the device and VE. The interface circuitry is the significant controlling part known as HIC, consist of a spring-damper model shown in Fig. 8 and given in (28).

$$
C(s)=K+\frac{B}{s}
$$

where $K$ is the virtual stiffness, $B$ is the virtual damping.

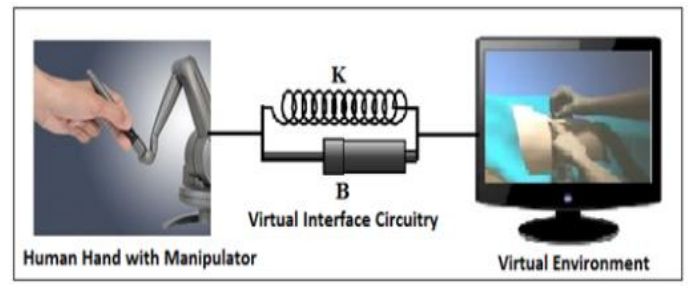

Fig. 8. Spring damper model of haptic system or HIC

The parameter for this model affects the system's stability and transparency. The parameters of the interface circuitry i.e. $K$ and $B$ cannot be chosen significantly high or low because at their high-values system can vibrate violently, and at the lower value, the system may not give an adequate response to user input. The limiting values (boundary values) of these parameters are given by Gil et al. [6] as (29), and (30) as below using the stability analysis by Routh-Hurwitz stability criterion (R-H) criterion.

$$
\begin{gathered}
K<\frac{1}{\frac{T}{2}+t_{d}}(b+B) \\
b+B>\frac{K T}{2}
\end{gathered}
$$

where $K$ is the virtual stiffness, $B$ is the virtual damping, $\mathrm{T}$ is sampling time period, time delay $t_{d}$, the physical damping $b$.

Moreover, to make system model more realistic, the unmodeled haptic system dynamics as transfer function $W(\mathrm{~s})$ and uncertainty as delta $\Delta$ varying in range $-1 \leq \Delta \leq 1$, are also incorporated while designing simulation model [32], [33]. The $W(\mathrm{~s})$ is modeled as

$$
W(s)=\psi m s+\Omega b
$$

The complete system model including uncertainty, delay, and quantization is shown in Fig. 9.

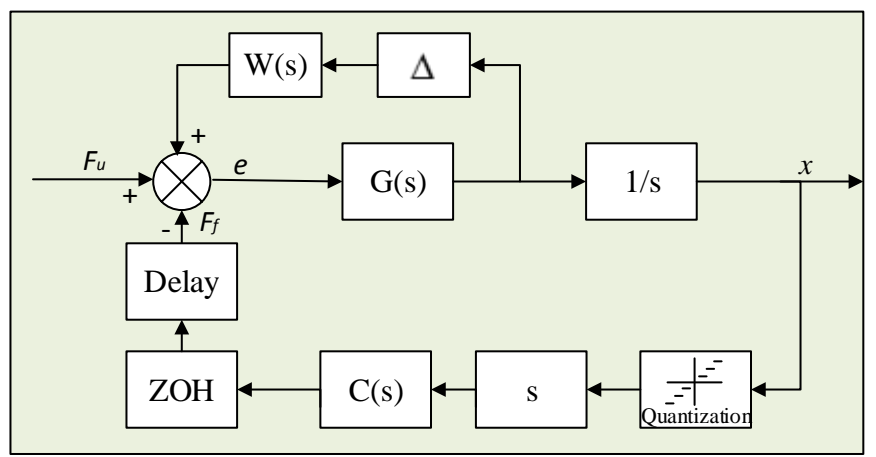

Fig. 9. Haptic system with uncertainty and delay

\section{HIC DESIGN AND Simulation RESUlts}

The objective of this work is to design the HIC controller $C(\mathrm{~s})$, to find out the optimal value of $K$ and $B$ satisfying the boundary values for stability condition so that the stability and transparency both are preserved, as both are complementary in nature to each other. The effectiveness of the proposed optimization technique presented in the previous section is tested in the critical optimal problem of haptic system to design HIC. The objective of the HIC controller is to maintain stability while increasing transparency. The error in the input force applied by the user (fixed as $5 \mathrm{~N}$ in this case) and the feedback force is to be minimized. So that system becomes stable as the oscillations (transient) should die as earliest and the user must experience the same force required from $\mathrm{VE}$ as the force applied by the user. It is also essential that feedback force and force error must settle down to steady-state as early as possible [34]. Hence to achieve this objective, it is of utmost important to optimally design the HIC controller. The objective of the intelligent techniques is to find the optimal values of the parameters of $K$ and $B$ so that both transparency and stability is achieved and the trade-off between them is satisfied.

\section{A. HIC Design using Neural Network}

In this section, a single hidden layer type $\mathrm{NN}$ has been proposed to use with five neurons hidden for better performance and easy computation for the design of HIC [14]. The training data set has been obtained from HIC controller design using $\mathrm{ZN}$ method [33] as given in Table 1. 
TABLE I

NN PARAMETERS TO DESIGN HIC

\begin{tabular}{ll}
\hline \hline Number of Layers & 2 \\
Number of Inputs & 1 \\
Number of Output & 1 \\
Number of Weight Elements & 10 \\
Activation Function & tansig \\
Transfer Function & traingda \\
\hline \hline
\end{tabular}

Following is the algorithm for FFNN:

Step 1: First, training data for $\mathrm{NN}$ has been prepared using $\mathrm{ZN}$ method. Here error is considered as input, and the control signal $u$ is considered as output.

Step 2: Now, train the NN using the feed-forward backpropagation method (FFNN).

Step 3: Simulate FFNN model with input as an error signal, and control parameters.

Step 4: Store the error signal as well as the control parameter obtained.

Step 5: Repeat step 3 to 4 till stopping criterion achieved.

Step 6: Pick the control parameter giving a minimum error signal and obtained force error, feedback force $\left(F_{f}\right)$ are shown in Fig 7 and 8 respectively.

Step 7: Simulate the Haptic system Model using obtained control parameters in step 6 and obtained error in force $\left(F_{\mathrm{f}}\right)$

Using this algorithm, simulation experiments is performed for design of HIC and results obtained as the error in force $e(t)$, and feedback force $\left(F_{\mathrm{f}}\right)$ are shown in Fig 10 and 11, respectively.

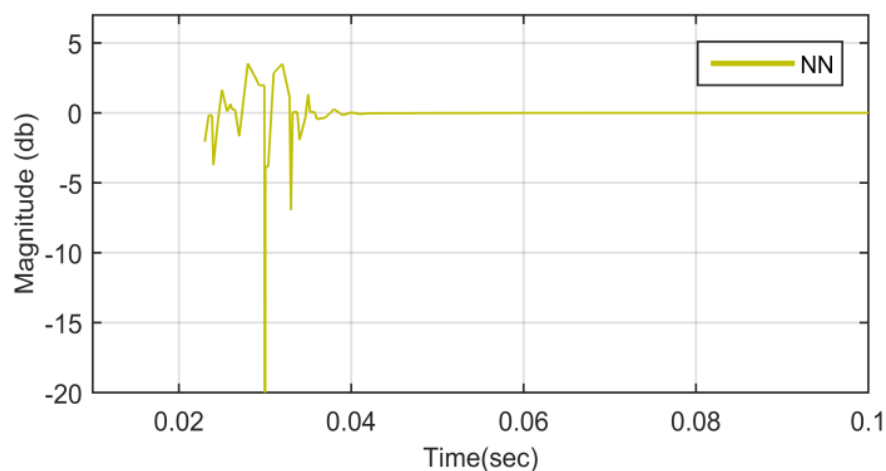

Fig. 10. Force error response $e(t)$ using neural network

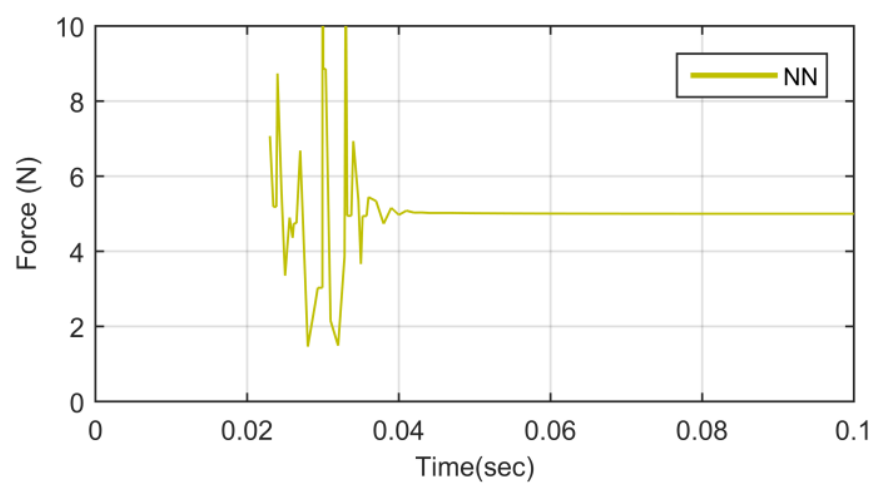

Fig. 11. Feedback Force $(F f)$ using neural network

Figures 10 and 11 show stable responses. The objective of the HIC controller is to maintain stability while increasing transparency. The error $e(t)$ in the force (input force $=5 \mathrm{~N}$ ), and the feedback force is to be minimized. It is also essential that feedback force and force error $e(t)$ must settle down as early as possible [34]. The oscillations in force error is settled down at $38 \mathrm{~ms}, K$ and $B$ obtained as 3.0083 and 301.8379 , respectively.

\section{B. HIC Design using Support Vector Machine}

The SVM optimizer has been used to find the optimal parameter of HIC. It uses two MATLAB libraries 'svmtrain' and 'svmpredict' have been used for SVM regression. The user format for these library has been given below:

Model= svmtrain (training_output, training_input, "libsvm options").

A pre-trained model has been used for the class of new input data.

Predict label $=$ svmpredict (testing output, testing input, "libsvm options").

Training data for SVM regression model has been obtained from the design of HIC using ZN method. The input data to the SVM is velocity and error. Using the said library, simulation experiments have been performed and the control parameters are obtained as $K=6.888$ and $B=1000$. The force error $e(t)$, force feedback error $F_{f}$ are shown in Fig 12 and 13, respectively.

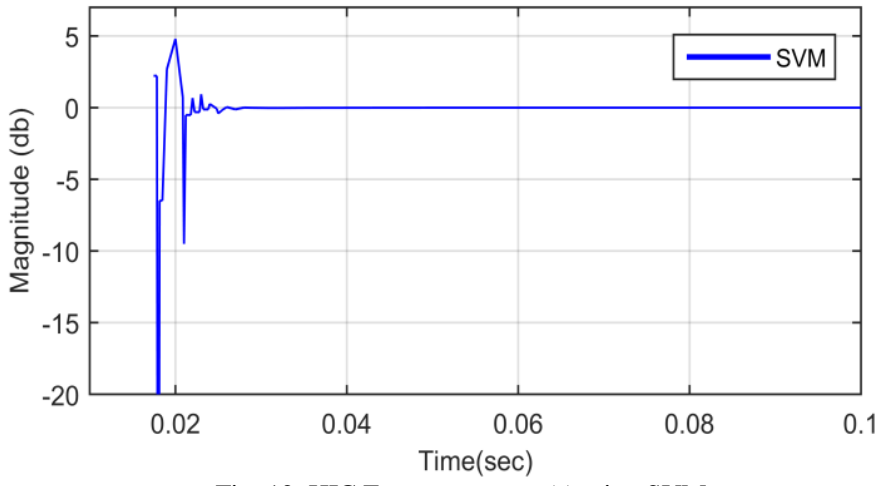

Fig. 12. HIC Error response $e(t)$ using SVM

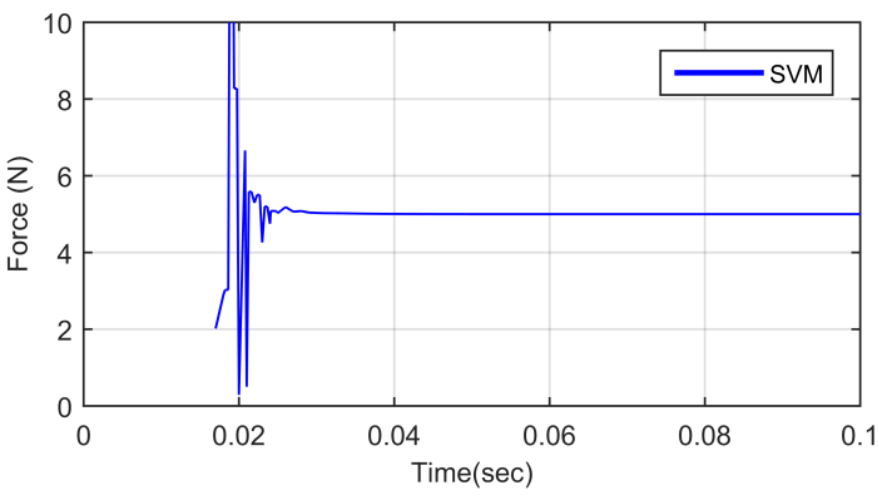

Fig.13. HIC Feedback Force $(F f)$ response using SVM

The results obtained using the proposed SVM based HIC design gives better results as compared to NN based HIC in terms of improved settling time and lesser initial oscillations. The minimization in settling time means the input signal is executed perfectly immediately as desired. These improvements in settling time has been shown in Fig 12 and 13. It has been observed that, when HIC parameter are designed using SVM, the settling time of force error signal has been 
reduced to $29 \mathrm{~ms}$, which is better than the conventional technique Z-N method (60 ms) and as well as for settling time obtained with Neural Network (38 ms).

Further, it is also observed by performing many simulation experiments that the choice of free parameters $(C, \gamma$, and $\varepsilon)$ greatly influences the performance of SVM. Also, these parameters are to be selected again if any change is made in the system or any uncertainty is added into the system. This selection process has to follow again for different applications also. To overcome this problem, an optimal tool is can be employed to select the SVM independent parameters.

\section{HIC Design using Proposed m-PSO Optimized SVM}

This section employs the proposed modified PSO optimized SVM to design the HIC for haptic system [35]. Here, m-PSO has been utilized to overcome the drawbacks of manual selection of SVM independent parameters. In m-PSO algorithm, first range of independent parameters, the cost factor $(C)$, gamma $\gamma$, and epsilon $(\varepsilon)$ of SVM is defined and initialized. Then fitness function is evaluated for the best performance of HIC with different combinations of SVM parameters. The mPSO algorithm is stopped when the best performance is achieved. Using this proposed technique for HIC design, the optimal parameters of HIC for $K$ and $B$ are 8.3541 and 800, respectively. Using these optimal value, output force error $e$ and feedback force response is shown in Fig. 14 and 15, respectively.

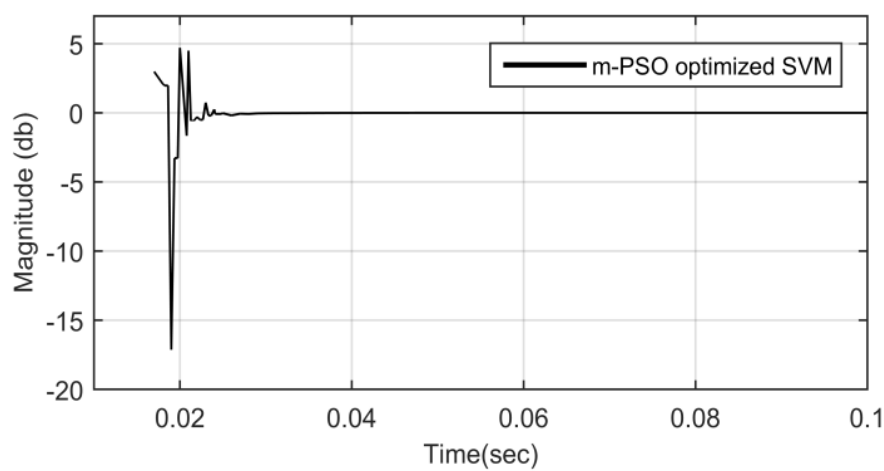

Fig. 14. Error response $e$ using m-PSO optimized SVM

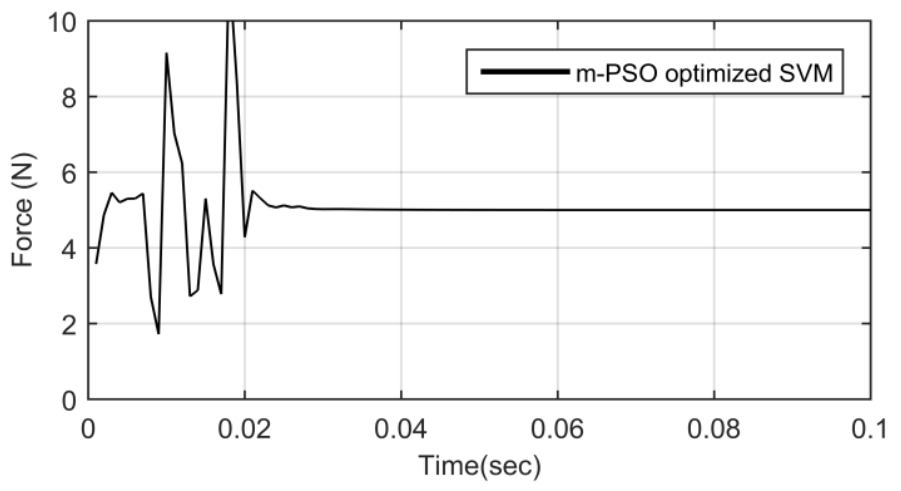

Fig. 15. Feedback Force response $(F f)$ using m-PSO optimized SVM

These figures show that the settling time and initial oscillations in output responses are reduced. The reduced oscillation at the initial phase means fewer vibrations in objects in Virtual Environment and in feedback force to the user also. Both of these are important requirements, for the haptic device applications in medical and other precise applications. The settling time for force error response is $24 \mathrm{~ms}$, which is least as compared to SVM, NN, and conventional ZN method.

\section{RESULTS AND DISCUSSION}

For the comparison purposes, the result obtained using proposed m-PSO optimized SVM is compared with SVM, NN, and conventional $\mathrm{ZN}$ method. The force error $e(t)$ and feedback force $F_{f}$ responses from all the above techniques are shown in Fig. 16 and 17, respectively.

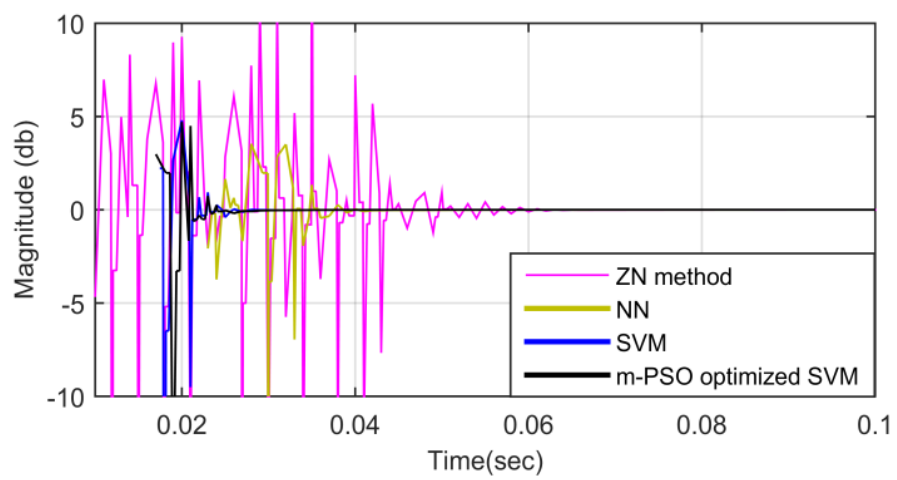

Fig. 16. Error response $e$ using m-PSO optimized SVM

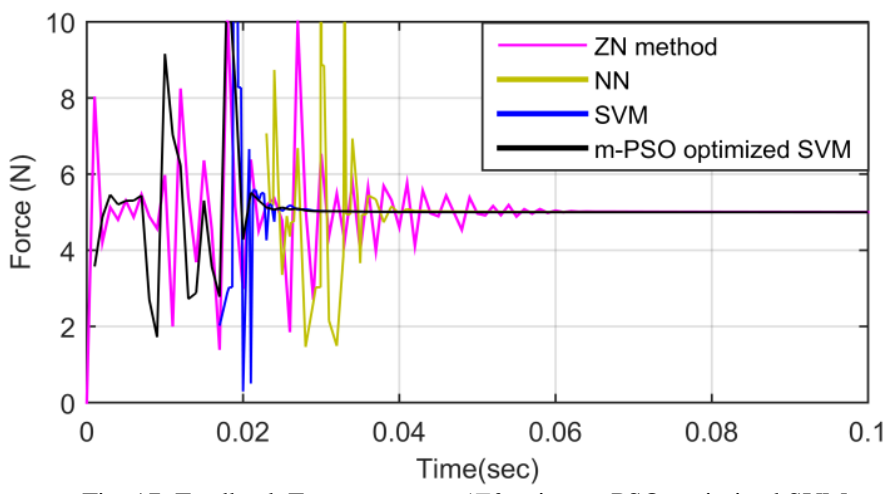

Fig. 17. Feedback Force response $(F f)$ using m-PSO optimized SVM

It has been observed from the said figures that HIC design using m-PSO optimized SVM gives better result among other discussed techniques in terms of reduced settling time and initial oscillations. The parameters of HIC, as well as the performance measure obtained with different design methods obtained in this work, are tabulated in Table II and Table III respectively.

TABLE II

PARAMETERS OF HIC WITH UNCERTAINTY AND DELAY

\begin{tabular}{|c|c|c|c|c|c|}
\hline $\begin{array}{c}\text { Controller } \\
\text { pethods }\end{array}$ & $\begin{array}{l}\text { Virtual } \\
\text { wall } \\
\text { dynamics }\end{array}$ & $\begin{array}{l}\mathrm{ZN} \\
\text { method }\end{array}$ & $\mathrm{NN}$ & SVM & $\begin{array}{c}\text { m-PSO } \\
\text { optimized } \\
\text { SVM }\end{array}$ \\
\hline $\begin{array}{l}\text { Virtual } \\
\text { stiffness }\end{array}$ & K & 17 & 3.0083 & 6.888 & 8.3541 \\
\hline $\begin{array}{l}\text { Virtual } \\
\text { damping }\end{array}$ & B & 800 & 301.8379 & 1000 & 800 \\
\hline
\end{tabular}

The performance measure given in Table III shows that, there is an improvement in settling time, shorter the peak overshoot, and 2-norms of error signal $e(t)$ are shown in the form of bar graph for pictorial comparison and analysis in Fig. 18, 19, 20 
and 21, respectively.

TABLE III

PERFORMANCE MEASURE OF HIC OBTAINED

\begin{tabular}{l|cccc}
\hline \hline \multirow{2}{*}{$\begin{array}{l}\text { Performance } \\
\text { Measure }\end{array}$} & $\begin{array}{l}\text { ZN } \\
\text { method }\end{array}$ & NN & SVM & $\begin{array}{c}\text { m-PSO } \\
\text { optimized } \\
\text { SVM }\end{array}$ \\
\cline { 2 - 5 } & & & & 24 \\
Settling Time & 60 & 38 & 5 & 5 \\
Peak overshoot & 21 & 4 & 61.144 & 19.682 \\
$\begin{array}{l}\text { 2-Norm of error } \\
e(t)\end{array}$ & 151.988 & 82.4901 & & 0.0016 \\
Mean(abs $(e))$ & 0.0391 & 0.0183 & 0.0154 & 0. \\
\hline
\end{tabular}

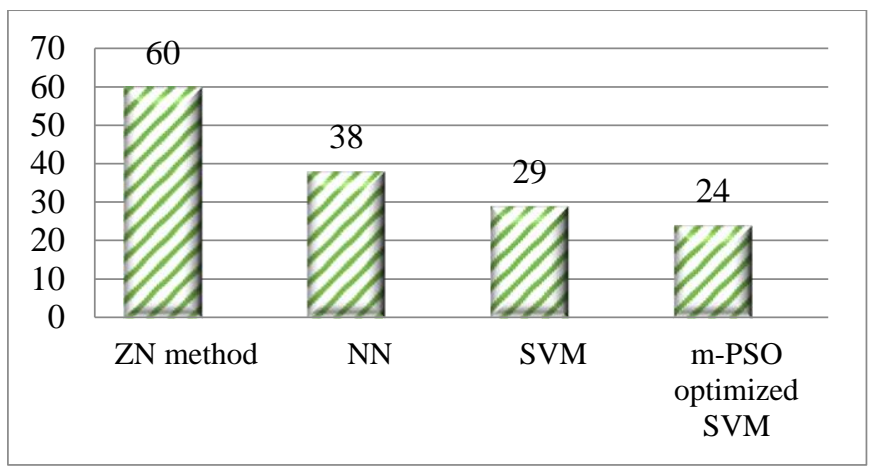

Fig. 18. Performance of HIC in terms of error $e(t)$ setting time (ms)

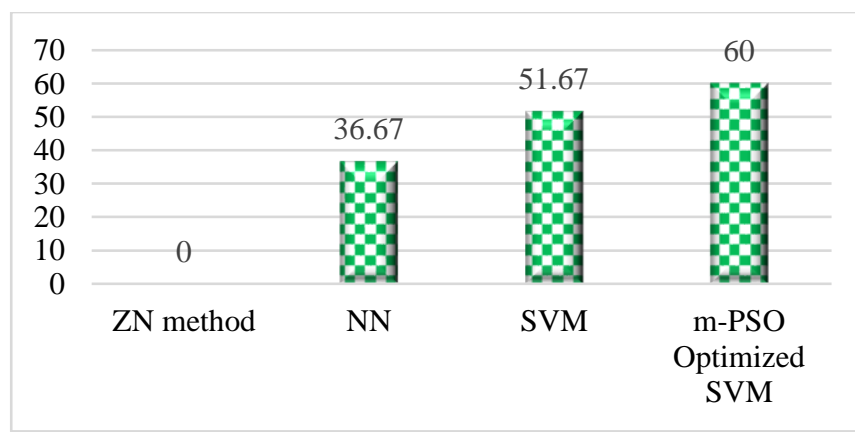

Fig. 19. Percentage improvement in settling time with different method

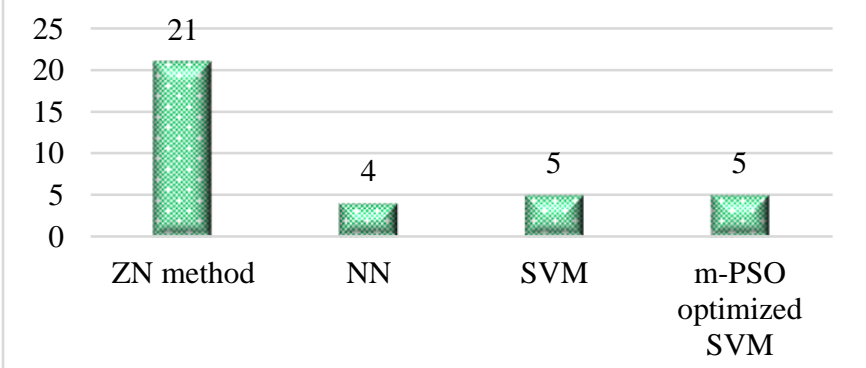

Fig. 20. Performance comparison of HIC in terms of Peak Overshoot

In Fig. 19, the improvement obtained in settling time of force error $e(t)$ with applied techniques is compared with $\mathrm{ZN}$ method is shown. It has been analyzed that there is $57 \%$ improvement in settling time in case of m-PSO optimized SVM technique as compared to $\mathrm{ZN}$ method. The 2-norm of the error and peak overshoot is also reduced up to much extent in the proposed method. Here it is worth mentioning that the m-PSO perform outstanding performance for optimizing the SVM model, and hence this proposed technique outperforms other techniques.

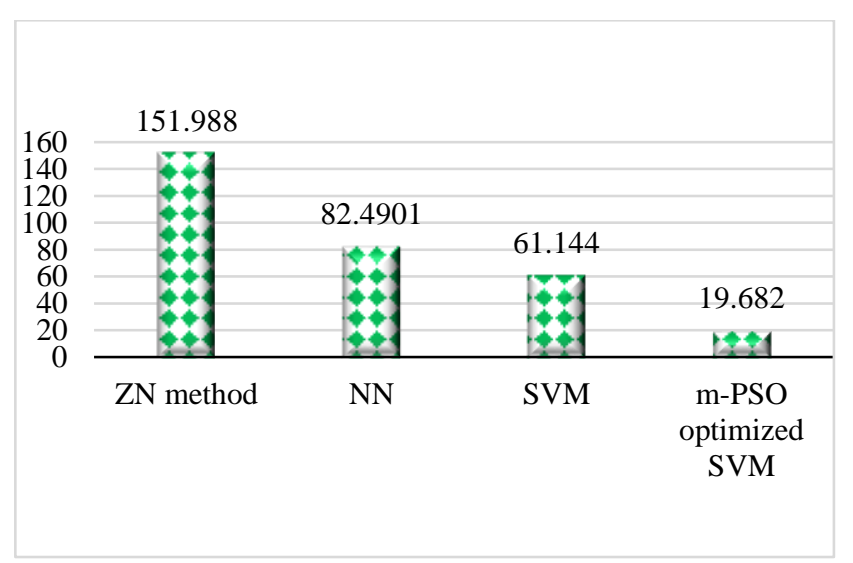

Fig. 21. Performance comparison of HIC in terms of 2-Norm of error

\section{CONCLUSION}

This paper presents a novel m-PSO optimized SVM technique for designing the haptic interface controller. A haptic system has performance issues in terms of stability and transparency, but both are complementary in nature to each other. When stability is enhanced, transparency gets hampered and vice-versa. The conventional $\mathrm{ZN}$ method is not able to find the optimal value to maintain the balance between two. To overcome this problem, intelligent techniques such as NN and SVM prove to be successful. NN disadvantages in terms of the number of hidden layers and neurons are conquered by SVM. Further, the selection of three independent parameters of SVM (cost factor (C), gamma $(\gamma)$, and epsilon $(\varepsilon)$ ), which significantly influence the performance of SVM. Hence, their optimal selection is up-most important to ensure the performance of SVM. The proposed modified PSO optimized SVM technique successfully ensures both the transparency and stability for HIC design. In this paper, m-PSO is employed for the optimal selection of free parameters of SVM. It has advantages in optimal selection of independent parameters over basic SVM. The performance of the proposed m-PSO optimized SVM is compared with SVM, NN, and conventional $\mathrm{ZN}$ method. Based on this, it has been observed that the proposed novel hybrid technique shows stable and better results in various performance measures. The lower the settling time higher the transparency, so this proposed technique results in an improvement in settling time over $\mathrm{ZN}$ method simultaneously ensuring lesser transient and average error.

\section{ACKNOWLEDGMENT}

The first author is thankful to UGC New Delhi, India, for financial support.

\section{REFERENCES}

[1] A. El Saddik, "The potential of haptics technologies," IEEE Instrum. Meas. Mag., vol. 10, no. 1, pp. 10-17, 2007, doi: 10.1109/MIM.2007.339540. 
[2] H. W. Kim, K. S. Eom, I. H. Suh, and B. J. Yi, "A transparencyoptimized control for a 6-DOF parallel-structured haptic device," in Proceedings of IEEE International Conference on Robotics and Automation, 2001, pp. 2331-2336, doi: 10.1109/ROBOT.2001.932970.

[3] D. Weir and J. Colgate, "Stability of Haptic Displays," in Haptic Rendering: Foundations, Algorithms, and Applications, 2008, pp. 123156.

[4] J. E. Colgate and G. G. Schenkel, "Passivity of a class of sampled-data systems: Application to haptic interfaces," J. Robot. Syst., vol. 14, no. 1, pp. 37-47, 1997, doi: 10.1002/(SICI) 1097-4563(199701)14:1<37::AIDROB4>3.0.CO;2-V

[5] J. E. Colgate and J. M. Brown, "Factors affecting the Z-Width of a haptic display," in Proceedings of the IEEE International Conference on Robotics and Automation, 1994, pp. 3205-3210, doi: 10.1109/ROBOT.1994.351077.

[6] J. J. Gil, A. Avello, ac A. Rubio, and J. Flórez, "Stability analysis of a 1 DOF haptic interface using the Routh-Hurwitz criterion," IEEE Trans. Control Syst. Technol., vol. 12, no. 4, pp. 583-588, 2004, doi 10.1109/TCST.2004.825134.

[7] K. S. Eom, I. H. Suh, and B.-J. Yi, "A design method of a haptic interface controller considering transparency and robust stability," in Proceedings of IEEE/RSJ International Conference on Intelligent Robots and Systems, 2000, vol. 2, pp. 961-966, doi: 10.1109/IROS.2000.893143.

[8] Y. Du, F. Shi, Q. Chen, Y. Wang, J. Zhao, and Q. Li, "An improved particle swarm scheduling algorithm based on batch changing production time," Eur. J. Electr. Eng., vol. 20, no. 4, pp. 439-453, 2018, doi: 10.3166/EJEE.20.439-453.

[9] M. Patrascu and A. Ion, Evolutionary Modeling of Industrial Plants and Design of PID Controllers, vol. 40. Cham: Springer International Publishing, 2016.

[10] A. Bashiti, M. Malkawi, M. A. Khasawneh, and O. Murad, "A novel neuro-fuzzy model to detect human emotions using different set of vital factors with performance index measure," J. Commun. Softw. Syst., vol. 14, no. 1, pp. 121-129, 2018, doi: 10.24138/jcomss.v14i1.392.

[11] A. Belkhou, A. Achmamad, and A. Jbari, "Myopathy detection and classification based on the continuous wavelet transform," J. Commun. Softw. Syst., vol. 15, no. 4, pp. 336-342, 2019, doi: 10.24138/jcomss.v15i4.796

[12] M. Sarayloo, E. Gambi, and S. Spinsante, "A new approach to sequence construction with good correlation by particle swarm optimization," $J$. Commun. Softw. Syst., vol. 11, no. 3, pp. 127-135, 2015, doi: 10.24138/jcomss.v11i3.101

[13] N. Kumar and J. Ohri, "LabVIEW based fuzzy logic controller for haptic interface," in Proceeding of IEEE 8th International Conference on Computing, Communications and Networking Technologies, ICCCNT 2017, 2017, pp. 1-6, doi: 10.1109/ICCCNT.2017.8204156.

[14] S. Surendran and V. Kumar, "Neural Network Based PI Controller Parameter Calculation on a Boiler Drum Level System," Procedia Technol., vol. 24, pp. 1616-1622, 2016, doi:10.1016/j.protcy.2016.05. 164.

[15] A. I. Glushchenko, "Neural tuner development method to adjust PIcontroller parameters on-line," in IEEE Conference of Russian Young Researchers in Electrical and Electronic Engineering (EIConRus), 2017 pp. 849-854, doi: 10.1109/EIConRus.2017.7910689.

[16] N. Kapoor and J. Ohri, "Evolutionary Optimized Neural Network (EONN) Based Motion Control of Manipulator," Int. J. Intell. Syst. Appl., vol. 6, no. 12, p. 10, 2014, doi: 10.5815/ijisa.2014.12.02.

[17] D. Boži-Stuli, S. Kruži, S. Gotovac, and V. Papi, "Complete model for automatic object detection and localisation on aerial images using convolutional neural networks," J. Commun. Softw. Syst., vol. 14, no. 1, pp. 82-90, 2018, doi: 10.24138/jcomss.v14i1.441.

[18] P. Janardhanan, L. Heena, and F. Sabika, "Effectiveness of support vector machines in medical data mining," J. Commun. Softw. Syst., vol. 11, no. 1, pp. 25-30, 2015, doi: 10.24138/jcomss.v11i1.114.

[19] E. L. Lasmar, R. L. Rosa, and D. Z. Rodríguez, "A recommendation system for shared-use mobility service through data extracted from online social networks," J. Commun. Softw. Syst., vol. 14, no. 4, pp. 359366, 2018, doi: 10.24138/jcomss.v14i4.602.

[20] E. Sariyildiz, K. Ucak, G. Oke, and H. Temeltas, "A Trajectory Tracking Application of Redundant Planar Robot Arm via Support Vector Machines," in Lecture Notes in Computer Science, vol. 6943, 2011, pp. 192-202.

[21] S. Jain and S. Kapshe, Modelling and Simulation using MATLABSimulink. Willey India Publications, 2016.

[22] V. Vapnik and A. Lerner, "Patern rrecocnition using generalized portralts," Avtomatatika i Telemekhanika, vol. 24, no. 6, pp. 774-780, 1963, doi: citeulike-article-id:619639.

[23] N. Kapoor and J. Ohri, "PD-SVM Integrated Controller for Robotic Manipulator Tracking Control," Chinese J. Eng., vol. 2014, 2014.

[24] A. Smola and B. Scholkopf, "A tutorial on support vector regression," Stat. Comput., vol. 14, no. 3, pp. 199-222, 2004, doi: 10.1023/B:STCO.0000035301.49549.88.

[25] H. . Wei and I. Cluckie, "Support vector machines identification for runoff modeling," in Proceedings of the 6th International Conference on Hydroinformatics, 2004, pp. 1597-1604.

[26] Y. B. Dibike, S. Velickov, D. Solomatine, and M. B. Abbott, "Model induction with support vector machines: Introduction and applications," J. Comput. Civ. Eng., vol. 15, no. 3, pp. 208-216, 2001, doi: 10.1061/(ASCE)0887-3801(2001)15:3(208)

[27] B. Chopard and M. Tomassini, "Particle swarm optimization," in Natural Computing Series, vol. 46, no. 10, 2018, pp. 97-102.

[28] Y. Shi and R. Eberhart, "A modified particle swarm optimizer," in IEEE International Conference on Evolutionary Computation Proceedings, IEEE World Congress on Computational Intelligence, 1998, pp. 69-73, doi: 10.1109/icec.1998.699146.

[29] D. Bratton and J. Kennedy, "Defining a standard for particle swarm optimization," in Proceedings of the IEEE Swarm Intelligence Symposium, SIS 2007, 2007, pp. 120-127, doi: 10.1109/SIS.2007.368035

[30] N. Kapoor and J. Ohri, "Improved PSO tuned Classical Controllers (PID and SMC) for Robotic Manipulator," Int. J. Mod. Educ. Comput. Sci., vol. 7, no. 1, pp. 47-54, 2015, doi: 10.5815/ijmecs.2015.01.07.

[31] P. C. Fourie and A. A. Groenwold, "The particle swarm optimization algorithm in size and shape optimization," Struct. Multidiscip. Optim., vol. 23 , no. 4, pp. 259-267, 2002, doi: 10.1007/s00158-002-0188-0.

[32] J. J. Gil, E. Sánchez, T. Hulin, C. Preusche, and G. Hirzinger, "Stability boundary for haptic rendering: Influence of damping and delay," in Proceedings - IEEE International Conference on Robotics and Automation, 2007, no. April, pp. 124-129, doi: 10.1109/ROBOT.2007.363775.

[33] N. Kumar and J. Ohri, "Design of Haptic Interface Controller under Noise Uncertainty and Delay Condition," Procedia Comput. Sci., vol. 70, pp. 793-800, 2015, doi: 10.1016/j.procs.2015.10.119.

[34] N. Enayati, E. De Momi, and G. Ferrigno, "Haptics in robot-assisted surgery: Challenges and benefits," IEEE Rev. Biomed. Eng., vol. 9, pp. 49-65, 2016, doi: 10.1109/RBME.2016.2538080.

[35] C. Wang, Y. Zhang, J. Song, Q. Liu, and H. Dong, “A novel optimized SVM algorithm based on PSO with saturation and mixed time-delays for classification of oil pipeline leak detection," Syst. Sci. Control Eng., vol. 7, no. 1, pp. 75-88, 2019, doi: 10.1080/21642583.2019.1573386.

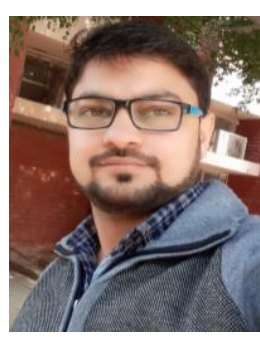

Naveen Kumar did his B.Tech from Kurukshetra University Kurukshetra and M.Tech from National Institute of Technology Kurukshetra (An institution of National Importance). Presently he is doing $\mathrm{PhD}$ from National Institute of Technology Kurukshetra. His area of interest includes robotic haptic control, robust and intelligent control etc. He has brief but significant teaching as well as research experience in his basket. He has various research articles in different Journals and Conferences. Mr. Kumar is member of various professional societies such as IEEE, IEEE Control System Society, IEEE Computer Society Technical Community on Haptics.

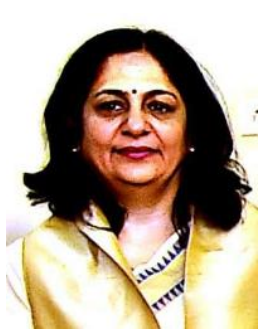

Dr. Jyoti Ohri did her B.Tech, M.Tech and PhD from National Institute Technology Kurukshetra, India. She is presently Professor in Electrical Engineering Department, National Institute Technology Kurukshetra, India. Her area of interest includes robotics, optimal robust and intelligent control etc. She has vast teaching and research experience to her account. She holds various research, academics and administrative position at different level. She have numerous research articles in various Journals and the Conferences. She is reviewer of various Journals. Prof. Ohri is member of various reputed professional societies such as IEEE, IEEE Control System Society, IEEE Computer Society Technical Community on Haptics. 